\title{
Multi-user Detection by MMSE Adaptive Algorithm for Multi-Beam-MIMO-MC-CDMA using Sequences of References
}

\author{
Mourad Benyarou \\ Abou-Bekr Belkaid University, \\ Faculty of Technology, \\ Telecommunications laboratory \\ P.O. Box 230, Tlemcen, Algeria
}

\author{
Fethi Tarik Bendimerad \\ Abou-Bekr Belkaid University, \\ Faculty of Technology, \\ Telecommunications laboratory \\ P.O. Box 230, Tlemcen, Algeria
}

\author{
Fatima Debbat \\ Mustapha stambouli University, \\ department of computer \\ science \\ P.O. Box 305,Mascara, Algeria
}

\begin{abstract}
The main objective of the multi-antenna technology is improving the capacity of cellular systems. These techniques can also make other improvements such as the scope of the system, reducing the transmission power of mobile (and therefore increasing energy autonomy) and improved link performance. This was the context of this paper study whose objective was to study the contribution and providing of the use of adaptive antennas in multiuser detection for MC-CDMA system. Transmission implicated in the MC-CDMA system has potential interference rejection but not sufficiently in the case of strong interference. Therefore it need to use beamforming algorithms. This work is also interested in MIMO-MC-CDMA with multiple-beamforming (MBF) at the receiver, that uses multiple antennas at transmitter and receiver. This paper presents also the performance of general MIMO-MC-CDMA system used Multi-Beamformer with sequences of references at receiver and space time block code (STBC) or vertical bell labs space-time architecture (V-BLAST) at the transmitter, with minimum mean square error (MMSE) adaptive algorithm under Rayleigh fading channels.
\end{abstract}

\section{General Terms.}

Multiplexing, diversity, beamforming, multicarrier,

performance

\section{Keywords:}

MC-CDMA, MIMO, VBLAST, STBC, Multiuser detection, Multi-beamforming, MMSE Adaptive Algorithm.

\section{INTRODUCTION}

The optimization of new transmission techniques which provide robustness and high spectral efficiency is crucial to the development of future cellular networks for radiocommunication. Like the success achieved in recent years by the multi-carrier modulation techniques which is based on OFDM and CDMA, the innovative techniques of transmission MC-CDMA are emerging today as candidate solutions for fourth generation cellular networks [1-2] with robustness potential of multi-carrier modulation on the one hand and flexibility in the sharing of radio resources for CDMA on the other hand.MC-CDMA techniques have improved significantly the ability of these future networks [3-4]. Meanwhile, another promising approach is to exploit spatial diversity or spatial multiplexing using multiple antennas at both the transmitter and the receiver, this approach is called MIMO systems.

The purpose of this paper is to study the association of MCCDMA and MIMO systems in order to exploit the spatial diversity or spatial multiplexing at transmitter with multi-beam at receiver. Multiuser detection is presented in the case of uplink for cellular systems.

\section{MC-CDMA SIGNAL}

In the MC-CDMA modulator shown in Figure 1, the data stream is first coded, interleaved and modulated into symbols $x$ by BICM block, and then the stream obtained is spread in the frequency domain using a spreading code and converted in substreams by the serial to parallel converter and transmitted on different sub carriers of the OFDM multiplex. A portion of each original data, corresponding to a chip of the spreading code with length $L_{c}$, is thus transmitted by each of the $N_{c}$ sub carriers.

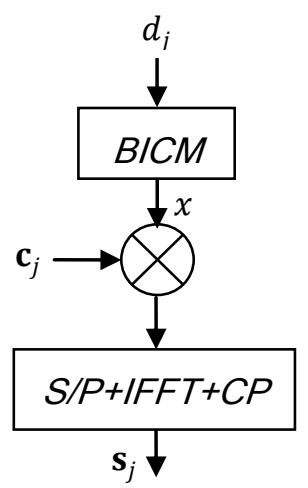

Fig1: Modulator MC-CDMA

In the case of a downlink where the various signals targeting different users are transmitted synchronously, the codes used are generally selected orthogonal, which results in receiving a better rejection of interference between users. Thus, with Walsh-Hadamard codes, the maximum number of users equals the number of codes. Generally, the number of $L_{c}$ chips of the spreading code is chosen equal to the number $N_{c}$ of subcarriers [5-6] but variants are possible to better adapt the signal to the channel in the case of an OFDM symbol by using an operation of inverse Fourier transform. 


\section{TRANSMITTER WITH MIMO MAPPING}

Figure 2 shows the block diagram of an MC-CDMA transmitter with MIMO mapping and Multi-beamforming at the receiver.

First, the data input $d_{j}$ for the $j^{\text {th }}$ user is modulated into a symbol $x$ with a BICM (Bit Interleaved Coded Modulation) encoder. Next, Mapping MIMO takes a block of modulated symbols and maps them into orthogonal sequences of length $L_{1}$

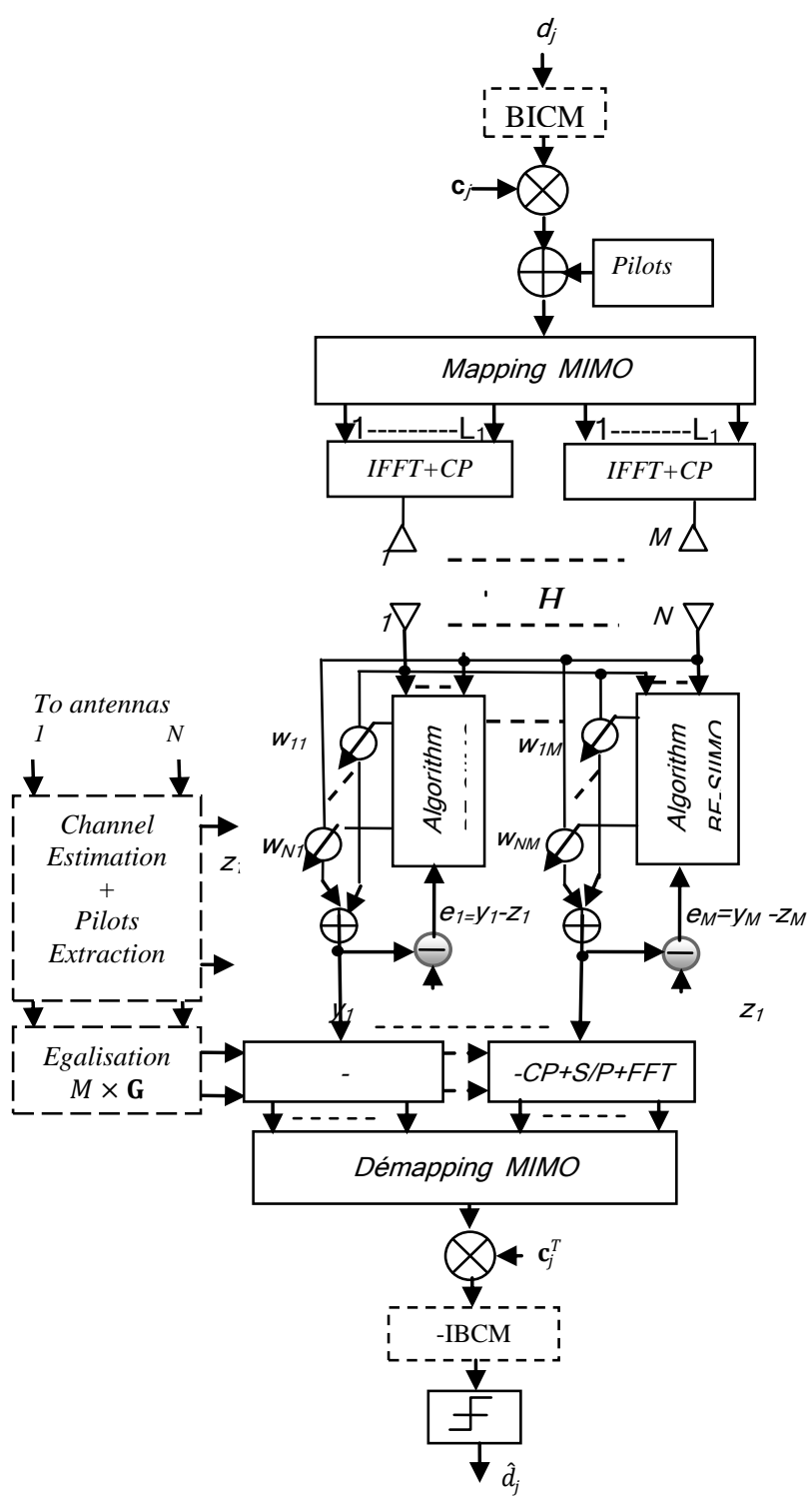

Fig 2: MBF-MIMO-MC-CDMA with spatial multiplexing or spatial diversity.

Let $\mathbf{x}_{i}$ be an $1 \times L_{1}$ symbol sequence for the $i^{\text {th }}$ transmit antenna given by

$\mathbf{x}_{i}=\left[x_{i 1, \ldots, x_{i L_{1}}}\right], i=1 \ldots, M$
Then for all antennas at transmitter it has

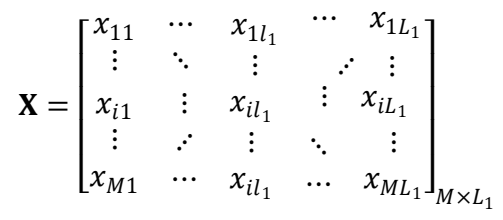

Where $M$ is denoted as the number of transmit antennas. Note that in the STBC case the orthogonality of the sequences enables to achieve the full transmit diversity for any number of transmit antenna and allows the receiver to decouple the signals transmitted from different antennas. Before transmitting, the MC-CDMA performs spreading, IFFT and cyclic prefix adding to avoid ISI as shown in Figure 2.

The radiated sequence can be modeled as

$$
\begin{gathered}
s_{i j l_{1}}(n)=\frac{1}{N_{c}} \sum_{p=0}^{N_{c}-1} \sqrt{\varepsilon_{j}} x_{i l_{1}} c_{j}(p) \exp \left(\frac{j 2 \pi p n}{N_{c}}\right) \\
n=0,1, \ldots, N_{c}-1
\end{gathered}
$$

Where $\mathbf{s}_{i j}(n)$ represents the $n^{\text {th }}$ sub channel sequence of the $j^{\text {th }}$ user that is transmitted by the $i^{\text {th }}$ antenna $; N_{c}=N_{e}=\frac{T_{s}}{T_{c}}=$ $L_{c} ; N_{c}, N_{e}, T_{s}, T_{c}$ and $L_{c}$ are respectively a number of subcarrier, the processing gain as a consequence of spreading ,time symbol ,time chip and code length,$c_{j}(p)$ is the $p^{\text {th }}$ chip of the code sequence and $\sqrt{\varepsilon_{j}}$ is the energy per symbol for the $j^{\text {th }}$ user.

In the MIMO channel, consider a scenario where there are $N_{U}$ users communicating synchronously with a common base station. Each user station (mobile station) has $M$ transmit antennas and the base station has $N$ receive antennas.

First we consider the Figure 3

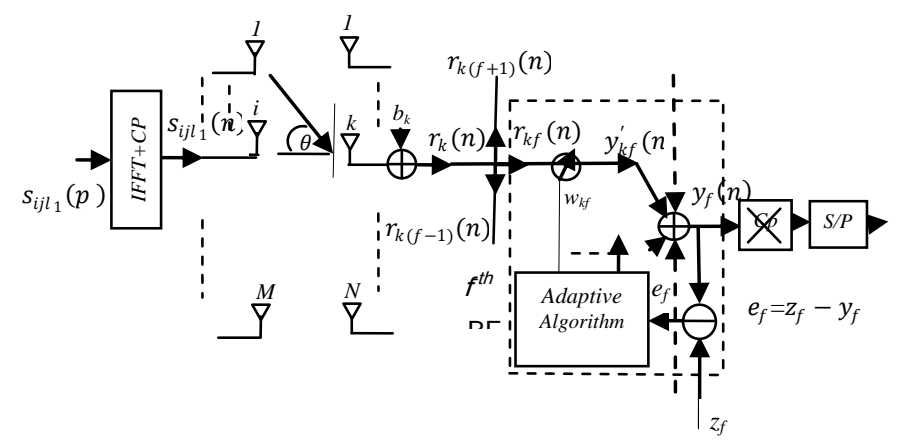

Fig 3: Principle illustrating the estimation of symbols 
The received signal from the $j^{\text {th }}$ user and $L$ propagation paths at the output of $k^{\text {th }}$ antenna is expressed as:

$$
\mathbf{r}_{j k}(n)=\sum_{i=1}^{M} a_{k}^{j}(\theta) \sum_{l=0}^{L-1} h_{k i}(n, l) \sum_{l_{1}=1}^{L_{1}} s_{i j l_{1}}(n-l)+\mathbf{n}(\mathrm{n})
$$

Where $s_{i j l_{1}}(\mathrm{n})$ is defined by (2) and $\sum_{l=0}^{L-1} h_{k, i, l} e^{\frac{-j 2 \pi l p}{N_{c}}}=$

$h_{k i, p}=\rho_{k i, p} e^{i \theta_{k i, p}}, p=0, \ldots, L_{c}-1$

$h_{k i, p}$ is the Fourier transform at element $h_{k, i, l}$ of the matrix of the MIMO multipath Channel for the $p^{\text {th }}$ sub carrier.

For all antennas reception at instant $n$ we have :

$$
\begin{aligned}
\mathbf{R}_{j}(n)=\sum_{k=1}^{N} \mathbf{r}_{j k}(n)= & \\
& {\left[\left(\mathbf{A}^{j} \odot \mathbf{H}^{p}\right) \mathbf{S}_{j}(n)+\mathbf{N}(n)\right]_{\left(\mathrm{N} \times L_{c}\right) \times\left(1 \times \mathrm{L}_{1}\right)} }
\end{aligned}
$$

$$
n=0,1, \ldots, N_{c}-1
$$

Where $\quad \mathbf{H}^{p}=\left[\sum_{k=1}^{N} \sum_{i=1}^{M} h_{k i, p}\right]_{(N \times 1) \times(M \times 1)}$ is the matrix channel for the $p^{\text {th }}$ sub carrier.

$$
\begin{gathered}
\mathbf{S}_{j}(n)=F F T^{-1}\left(\sqrt{\varepsilon_{j}} \mathbf{X} c_{j}(p)\right) \\
\mathbf{S}_{j}(n)=\left[\mathbf{s}_{1 j 1}(n) \cdots \mathbf{s}_{i j} l_{1}(n) \cdots \mathbf{s}_{M j L_{1}}(n)\right]_{M \times L_{1}}^{T} \\
\mathbf{X}_{c_{j}}(p)=\left[\begin{array}{ccccc}
x_{11} c_{j}(p) & \cdots & x_{1 l_{1}} c_{j}(p) & \cdots & x_{1 L_{1}} c_{j}(p) \\
\vdots & \ddots & \vdots & & \vdots \\
x_{i 1} c_{j}(p) & \vdots & x_{i l_{1}} c_{j}(p) & \vdots & x_{i L_{1}} c_{j}(p) \\
\vdots & \ddots & \vdots & \ddots & \vdots \\
x_{M 1} c_{j}(p) & \cdots & x_{i l_{1}} c_{j}(p) & \cdots & x_{M L_{1}} c_{j}(p)
\end{array}\right]_{M \times L_{1}}
\end{gathered}
$$

* The output for all antennas and all users at base station is given by

$$
\begin{gathered}
\mathbf{R}(n)=\sum_{j=1}^{N_{U}} \mathbf{R}_{j}(n)=\left[\mathbf{r}_{1}(n) \cdots \mathbf{r}_{N}(n)\right]_{(\mathrm{N} \times 1) \times\left(\mathrm{L}_{1} \times 1\right)}^{T} \\
=\left(\mathbf{A}(\theta) \odot \mathbf{H}^{p}\right) \mathbf{S}(n)+\mathbf{N}(n) \\
\mathbf{S}(n)=\sum_{j=1}^{N_{U}} \mathbf{S}_{j}(n)=\mathrm{FFT}^{-1}(\sqrt{\boldsymbol{\varepsilon}} \mathbf{X c}(p))
\end{gathered}
$$

$$
\mathbf{X c}(p)=\left[\begin{array}{ccccc}
x_{11} \mathbf{c}(p) & \cdots & x_{1 l_{1}} \mathbf{c}(p) & \cdots & x_{1 L_{1}} \mathbf{c}(p) \\
\vdots & \ddots & \vdots & & \vdots \\
x_{i 1} \mathbf{c}(p) & \vdots & x_{i l_{1}} \mathbf{c}(p) & \vdots & x_{i L_{1}} \mathbf{c}(p) \\
\vdots & \ddots & \vdots & \ddots & \vdots \\
x_{M 1} \mathbf{c}(p) & \cdots & x_{i l_{1}} \mathbf{c}(p) & \cdots & x_{M L_{1}} \mathbf{c}(p)
\end{array}\right]_{M \times L_{1}}
$$

$\mathbf{c}(p)=\left[\begin{array}{lllll}c_{1}(p) & \cdots & c_{j}(p) & \cdots & \left.c_{N_{U}}(p)\right]_{1 \times N_{U}}\end{array}\right.$

and $x_{i l_{1}} \mathbf{c}(p)=\sum_{j=1}^{N_{U}} x_{i l_{1}} \mathrm{c}_{j}(p)$

$\mathbf{A}^{j}(\theta)=\left[\mathbf{a}\left(\theta_{1 j}\right) \mathbf{a}\left(\theta_{2 j}\right) \cdots \mathbf{a}\left(\theta_{M j}\right)\right]_{N \times M}$

$\mathbf{a}\left(\boldsymbol{\theta}_{\boldsymbol{i j}}\right)=\left[1 e^{j 2 \pi \frac{d}{\lambda} \sin \theta_{i j}} \cdots e^{j 2 \pi \frac{d}{\lambda}(N-1) \sin \theta_{i j}}\right]_{N \times 1}^{T}$

$\mathbf{N}(n)=\mathbf{B}(n)+\mathbf{I}(n)$

$\mathbf{B}(n)=\left[\mathbf{b}_{1}(n) \mathbf{b}_{2}(n) \cdots \mathbf{b}_{N}(n)\right]_{(N \times 1) \times\left(L_{1} \times 1\right)}^{T}$

$\mathbf{I}(n)=\left[i_{1}(n) i_{2}(n) \cdots i_{N}(n)\right]_{N \times 1}^{T}$

$\odot$ is an operator of multiplying element by element or called Hadamard product. In the steering vector $\mathbf{a}\left(\boldsymbol{\theta}_{\boldsymbol{i}}\right), d$ is the interelement antenna spacing; $\lambda \square$ is the wavelength and $\theta_{i j}$ is the direction of arrival (DOA) of the $j^{\text {th }}$ user coming from the $i^{t h}$ antenna as shown in Figure 3. The matrix $\mathbf{H}_{(N \times 1) \times(M \times 1)}$ is a channel coefficient matrix for all paths. The coefficient amplitude is determined by the Rayleigh random variable while its phase is uniformly distributed in the interval $[0-2 \pi] . \mathbf{B}(n)$ is temporally and spatially uncorrelated Gaussian noise vector with zero mean and complex variance $\sigma_{b}^{2}$ and $\mathbf{I}(n)$ is the interference vector on all antennas reception.

It assumed that each path of each user incidents on the receive antenna array arrives with the same DOAs.

Note that by removing the cyclic prefix at the beginning of each received data block, the inter-symbol OFDM interference can be eliminated.

* For all users and for $0 \leq p \leq\left(N_{c}=L_{c}\right)$ and $0 \leq n \leq L_{c} \quad \mathbf{R}$ is given by

$\mathbf{R}=[(\mathbf{A}(\theta) \odot \mathbf{H}) \mathbf{S}+\mathbf{N}]_{\left(\mathrm{N} \times L_{c}\right) \times\left(\mathrm{L}_{1} \times L_{c}\right)}$

$\mathbf{S}=\left[F F T^{-1}\left(\sqrt{\varepsilon_{j}} \mathbf{X C}\right)\right]_{\left(M \times L_{c}\right) \times\left(L_{1} \times L_{c}\right)}$

$\mathbf{X C}=\left[\begin{array}{ccccc}x_{11} \mathbf{C} & \cdots & x_{1 l_{1}} \mathbf{C} & \cdots & x_{1 L_{1}} \mathbf{C} \\ \vdots & \ddots & \vdots & & \vdots \\ x_{i 1} \mathbf{C} & \vdots & x_{i l_{1}} \mathbf{C} & \vdots & x_{i L_{1}} \mathbf{C} \\ \vdots & \ddots & \vdots & \ddots & \vdots \\ x_{M 1} \mathbf{C} & \cdots & x_{i l_{1}} \mathbf{C} & \cdots & x_{M L_{1}} \mathbf{C}\end{array}\right]_{M \times L_{1}}$ 


$$
\begin{aligned}
& \mathbf{C}=\left[\mathbf{c}_{0} \cdots \mathbf{c}_{j} \cdots \mathbf{c}_{N_{U-1}}\right]_{L_{c} \times N_{U}} \\
& x_{i l_{1}} \mathbf{C}=\sum_{j=1}^{N_{U}} x_{i l_{1}} \mathbf{c}_{j} \\
& \mathbf{c}_{j}=\left[c_{j}(0) \cdots c_{j}(p) \cdots c_{j}\left(L_{c}-1\right)\right]_{L_{c} \times 1}^{T} \\
& \mathbf{H}=\left[\sum_{k=1}^{N} \sum_{i=1}^{M} \mathbf{H}_{k i}\right]_{\left(N \times L_{c}\right) \times\left(M \times L_{c}\right)}
\end{aligned}
$$

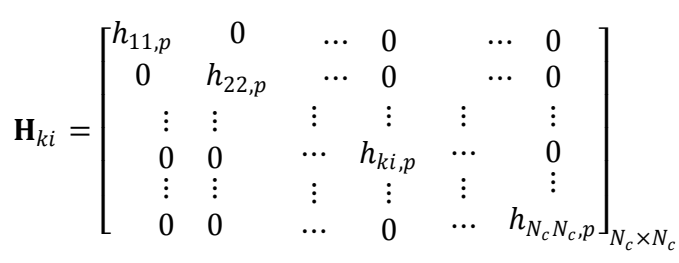

$\mathbf{H}_{k i}$ is a diagonal matrix of size $L_{c} \times L_{c}$ where each diagonal element corresponding to the channel frequency response at the subcarrier $p, h_{k i, p}=\rho_{k i p} e^{i \theta_{k i, p}} \quad$ whose coefficient $\rho_{k i p}$ is a magnitude determined by a random variable Rayleigh and $\theta_{k i, p}$ is the phase uniformly distributed in the interval $[0-2 \pi]$.

$$
\begin{aligned}
& \mathbf{N}=\mathbf{B}+\mathbf{I} \\
& \mathbf{B}=\left[\mathbf{B}_{1} \mathbf{B}_{2} \cdots \mathbf{B}_{N}\right]_{\left(N \times N_{c}\right) \times\left(L_{1} \times N_{c}\right)}^{T} \\
& \mathbf{I}=\left[i_{1} i_{2} \cdots i_{N}\right]_{N \times 1}^{T}
\end{aligned}
$$

The reception matrix $\mathbf{R}$ consists of $l_{1}$ columns $\mathbf{V}_{l_{1}}$. The columns $\mathbf{V}_{l_{1}}$ can be rewritten in time series as:

$$
\mathbf{V}_{l_{1}}=\left[\mathbf{V}_{l_{1}}(0) \cdots \mathbf{V}_{l_{1}}(n) \cdots \mathbf{V}_{l_{1}}\left(L_{c}-1\right)\right]_{\left(\mathrm{N} \times L_{c}\right) \times\left(1 \times L_{c}\right)}
$$

For despreading the received data, each column vector

$\mathbf{V}_{l_{1}} l_{1}=0, \ldots, L_{1}-1$ is multiplied by a Fourier matrix $\mathrm{F}$, then the result give:

$\mathbb{X}_{l_{1}\left(N \times L_{c}\right)}=\mathbf{V}_{l_{1}} \mathbf{F}_{L_{c} \times L_{c}}$

Where $\mathbf{F}_{L_{c} \times L_{c}}$ is the FFT and is given as

$$
\mathbf{F}=\frac{1}{L_{c}}\left[\begin{array}{cccc}
1 & 1 & \cdots & 1 \\
1 & e^{-j 2 \pi(1)(1) / L_{c}} & \cdots & e^{-j 2 \pi(1)\left(L_{c}-1\right) / L_{c}} \\
\vdots & \vdots & \ddots & \vdots \\
1 & e^{-j 2 \pi\left(L_{c}-1\right)(1) / L_{c}} & \cdots & e^{-j 2 \pi\left(L_{c}-1\right)\left(L_{c}-1\right) / L_{c}}
\end{array}\right]_{L_{c} \times L_{c}}
$$

Then multiplied each row of $\mathbb{X}_{l_{1}}$ by the code sequence give:

$$
\widehat{\mathbb{X}}_{j l_{1}}=\operatorname{row}\left(\mathbb{X}_{l_{1}}\right) \odot \mathbf{c}_{j}^{T}
$$

The method and the proposed adaptive beamforming are explained in the following subsections
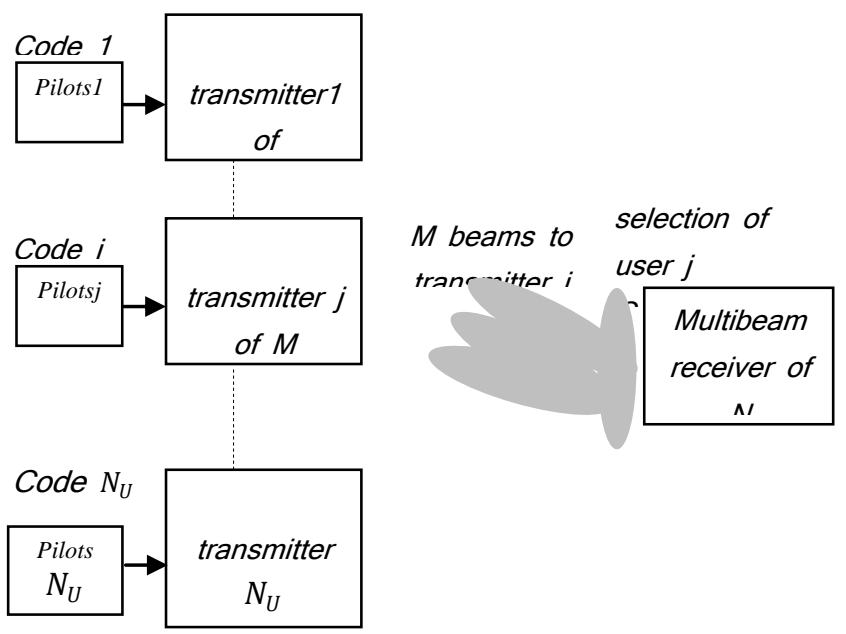

Fig 4 : Multiuser detection with multibeam systems

\subsection{CDMA case}

In the CDMA case the same equations are obtained but it need to omit the IFFT operation in eq (2) and IFFT /FFT in Figure 2 and replacing eq (2) by (15) and the $\mathbf{H}$ matrix is replaced by an multipath channel with $(N \times L) \times M$ dimension, $L$ is the number of paths:

$$
s_{i j l_{1}}(n)=\sqrt{\varepsilon_{j}} x_{i l_{1}} c_{j}(p)
$$

After, this equation is used for all the following.

\section{BEAMFORMING ALGORITHM}

This paper presents the multiuser detection with multibeam. The $M$ beams of the base station is directed to the $M$ antennas of each mobile as illustrated in Figure 4

Our adaptive algorithm is an MMSE algorithm with signal reference (pilot).This pilots are inserted for each antennas at transmitter. This adaptive algorithm is used for all Beamformer at receiver.

The error signal as chow on figure 3 is given by [7-8-9]

$e_{f}(n)=y_{f}(n)-z_{f}(n)$

(16)

The index $f$ is for the $f^{\text {th }}$ beamformer (see Figure 2 or 3 ) and $y_{f}(n)$ is the estimated of $s_{i j l_{1} i}(n)$ because in this case the beam $f$ is directed to the transmitting antenna $i$ for user $j$.

$$
\mathbf{w}_{j f}=\mathbf{w}_{S B_{f}}=\left[w_{j f 1} \cdots w_{j f k} \cdots w_{j f N}\right]_{N \times 1}^{T}, k=1, \cdots, N
$$


is called a Simple Beam weight vector number $f$ corresponding for user $j$ and $N$ is the number of antennas at the reception.

The weights of the vector $\mathbf{w}_{j f}$ are calculated according to the reference signal $z_{f}\left(\Leftrightarrow z_{i}\right.$ which is multiplexed with the $i^{\text {-th }}$ IFFT and injected into the transmitter antenna $i$ of the $j^{\text {th }}$ user).

Figure 3 is a subassembly of Figure 2, ie to allow the detection of only one symbol $s_{i j l_{1}}(n)$ by the beamformer $f$ at time $n$, then to detect all symbols $y_{f}, f=1, \cdots, M, M$ equations are obtained of the form:

$\left\{\begin{array}{cc}y_{1}(n)=\sum_{k=1}^{N} w_{j k 1}^{*} & r_{j 1}(n-k) \text { is the estimated of } s_{1 j l_{1}}(n) \\ y_{2}(n)=\sum_{k=1}^{N} w_{j k 2}^{*} & r_{j 2}(n-k) \text { is the estimated of } s_{2 j l_{1}}(n) \\ \vdots & \\ y_{f}(n)=\sum_{k=1}^{N} w_{j k f}^{*} & r_{j f}(n-k) \text { is the estimated of } s_{i j l_{1}}(n) \\ \vdots & \\ y_{M}(n)=\sum_{k=1}^{N} w_{j k M}^{*} & r_{j M}(n-k) \text { is the estimated of } s_{M j l_{1}}(n)\end{array}\right.$

Then for index $i$,

$\hat{s}_{i j l_{1}}(n)=y_{f}(n)=\sum_{k=1}^{N} w_{j k f} r_{j f}(n-k)=\mathbf{w}_{j f}^{H} \mathbf{r}_{j f}(n)$

Then for all symbols (or for all $M$ antennas) and for all users at time $n$ :

$\widehat{\mathbf{S}}(n)=\left[\mathbf{W}_{M B M U}^{H} \mathbf{R}(n)\right]_{M \times L_{1}}$

The formation of multiple beams is used to eliminate interferences on antennas array at reception, in neglecting the noise $\mathbf{B}(\mathrm{n})$ for simplifying the previous equation can be written as:

$\widehat{\mathbf{S}}(n)=\left[\mathbf{W}_{M B M U}^{H} \mathbf{A}(\theta) \odot \mathbf{H S}(n)\right]_{M \times L_{1}}$

$\mathbf{W}_{M B M U}$ is called Multi-Beam Multi-User weighting matrix and $\mathbf{S}(n)$ is defined by (8).

$-j$ is omitted for all next equations for convenience.

In the MMSE approach, the cost function to minimized is

$\boldsymbol{J}\left(w_{f}\right)=\mathrm{E}\left[\left|\mathbf{w}_{f}^{H} \mathbf{r}_{f}(n)-z_{f}(n)\right|^{2}\right]$

Where $z_{f}(n)=z_{f}\left(n T_{s}\right)$ and $\mathbf{r}_{f}(n)=\mathbf{r}_{f}\left(n T_{s}\right)$ where $T_{s}$ is the sampling period.

The cost function is the expected value (taken over the ensemble of realizations of $\mathbf{r}_{f}(n)$ ) of the square error between the array output and the desired version of that signal at time index $n$. (18) can be rewrite as :

$\boldsymbol{J}\left(w_{f}\right)=\mathbf{w}_{f}^{H} \mathrm{E}\left[\mathbf{r}_{f}(n) \mathbf{r}_{f}^{H}(n)\right] \mathbf{w}_{f}-\mathrm{E}\left[z_{f}(n) \mathbf{r}_{f}^{H}(n)\right] \mathbf{w}_{f}-$

$\mathbf{w}_{f}^{H} \mathrm{E}\left[\mathbf{r}_{f}(n) z_{f}^{*}(n)\right]+\mathrm{E}\left[z_{f}(n) z_{f}^{*}(n)\right]$

In general, the vector function is minimized by determining a location where the gradient of the function goes to zero, then the solution is given by :

$\nabla J\left(w_{f}\right)=2 \mathbf{R} \mathbf{w}_{f}-2 \mathbf{r}_{r s_{i}}$

Where $\mathbf{R}$ is the correlation matrix of the data vector

$\mathbf{R}=\mathrm{E}\left[\mathbf{r}(n) \mathbf{r}^{H}(n)\right]$

and $\mathbf{r}_{r s_{i}}$ is the cross-correlation vector between the data vector and the desired signal,

$\mathbf{r}_{r s_{i}}=\mathrm{E}\left[\mathbf{r}(n) s_{i}^{H}(n)\right]$

Setting the gradient of the cost function equal to zero, the solution for $\mathbf{w}_{f}$ wich minimizes $\boldsymbol{J}\left(w_{f}\right)$ is given by

$\mathbf{w}_{f}=\mathbf{R}^{-1} \mathbf{r}_{r s_{i}}$

The first step is to realize a deterministic iterative procedure to compute $\mathbf{w}_{f \text { opt }}$.It can see this method avoids the computation of the inverse $\mathbf{R}^{-1}$.

For this problem the steepest descent or gradient algorithm methods is used and is defined by,

$\mathbf{w}_{f}(k+1)=\mathbf{w}_{f}(k)-\frac{1}{2} \mu \nabla_{\mathbf{w}_{f}(k)} \boldsymbol{J}(k)$

Where $\nabla_{w_{f}(k)}$ denotes the gradient of $\boldsymbol{J}(k)$ with respect to $\mathbf{w}_{f}, \mu$ is the adaptation gain ,a real valued positive constant , and $k$ is the iteration index, in general not necessarily coinciding with time instants.

As $J(k)$ is a quadratic function of the vector coefficients, the solution is given by:

$\nabla_{\mathbf{w}_{f}(k)} J(k)=2\left(\mathbf{R w}_{f}(k)-\mathbf{r}_{r s_{i}}\right)$

hence

$\mathbf{w}_{f}(k+1)=\mathbf{w}_{f}(k)-\mu\left(\mathbf{R w}_{f}(k)-\mathbf{r}_{r s_{i}}\right)$ 
In the scalar case, for real-valued signals in [7] page $171 \mu_{\text {opt }}$ is defined as $\mu_{\text {opt }}=\frac{2}{\lambda_{\max }+\lambda_{\min }}$ where $\lambda_{\max }$ and $\lambda_{\min }$ are the eigenvalues of $\boldsymbol{\Lambda}$ when using decomposition $\mathbf{R}=\mathbf{U} \boldsymbol{\Lambda} \mathbf{U}^{\mathrm{H}}$ and $\mathbf{U}$ is the unitary matrix formed of eigenvectors of $\mathbf{R}$.

\section{SIMULATION RESULTS}

The performance of the proposed algorithm is evaluated by simulating BERs (bit error rates).

Figure 2 is considered, two antenna arrays are uniform and linear with a half-wavelength inter-element spacing, $M$ elements at the transmitter and $N$ elements at the receiver.The number of users is $N_{U}=4$. Each user is assigned 8 paths and come with almost the same angle incidence $\theta$.

The signal to noise ratio of Co-channel interference is low compared to that of the signal of interest and is uniformly distributed in the interval $[0,15] \mathrm{dB}$ and their directions are uniformly distributed in the interval [-70.70]. To generate the spreading codes the Hadamard matrix is used, the number of chips $L_{c}$ is equal to the number of subcarrier $N_{c}=$ 128.

To generate the pilot sequence $z_{f}, f=1, \cdots, M$ of each beamformer one OFDM symbol is inserted in each frame of the IFFT for MC-CDMA systems. The guard interval is selected equal to one quarter of the OFDM symbol duration.

In the MC-CDMA case the number of OFDM symbol in the frame is selected as a function of the length of the sequence of the $\mathbf{S}$ matrix and is equal to $N_{f_{r}} \geq\left(L_{1}+1\right), L_{1}$ is the length of the sequence of the $\mathbf{S}$ matrix, 1 represents an OFDM symbol of the reference sequence.

In the CDMA case after each sequence of the matrix $\mathbf{S}$ a PN sequence is used as a reference signal.

Spatial diversity is considered with two matrix $\mathbf{S}_{1}$ or $\mathbf{S}_{2}$ for STBC encoder and spatial multiplexing with two matrix $\mathbf{S}_{3}$ or $\mathbf{S}_{4}$ for VBLASTencoder.

Bpsk is used for the STBC modulation case and 16 QAM in the VBLAST case.

$$
\begin{gathered}
\mathbf{S}_{1}=\left[\begin{array}{cccccccc}
s_{1} & -s_{2} & -s_{3} & -s_{4} & s_{1}^{*} & -s_{2}^{*} & -s_{3}^{*} & -s_{4}^{*} \\
s_{2} & s_{1} & s_{4} & -s_{3} & s_{2}^{*} & s_{1}^{*} & s_{4}^{*} & -s_{3}^{*} \\
s_{3} & -s_{4} & s_{1} & s_{2} & s_{3}^{*} & -s_{4}^{*} & s_{1}^{*} & s_{2}^{*}
\end{array}\right]_{3 \times 8} \\
\mathbf{S}_{2}=\left[\begin{array}{cccccccc}
s_{1} & -s_{2} & -s_{3} & -s_{4} & s_{1}^{*} & -s_{2}^{*} & -s_{3}^{*} & -s_{4}^{*} \\
s_{2} & s_{1} & s_{4} & -s_{3} & s_{2}^{*} & s_{1}^{*} & s_{4}^{*} & -s_{3}^{*} \\
s_{3} & -s_{4} & s_{1} & s_{2} & s_{3}^{*} & -s_{4}^{*} & s_{1}^{*} & s_{2}^{*} \\
s_{4} & s_{3} & -s_{2} & s_{1} & s_{4}^{*} & s_{3}^{*} & -s_{2}^{*} & s_{1}^{*}
\end{array}\right]_{4 \times 8} \\
\mathbf{S}_{3}=\left[\begin{array}{lllllllll}
\mathrm{s}_{1} & \mathrm{~s}_{4} & \mathrm{~s}_{7} & \mathrm{~s}_{10} & \mathrm{~s}_{13} & \mathrm{~s}_{16} & \mathrm{~s}_{19} & \mathrm{~s}_{22} \\
\mathrm{~s}_{2} & \mathrm{~s}_{5} & \mathrm{~s}_{8} & \mathrm{~s}_{11} & \mathrm{~s}_{14} & \mathrm{~s}_{17} & \mathrm{~s}_{20} & \mathrm{~s}_{23} \\
\mathrm{~s}_{3} & \mathrm{~s}_{6} & \mathrm{~s}_{9} & \mathrm{~s}_{12} & \mathrm{~s}_{15} & \mathrm{~s}_{18} & \mathrm{~s}_{21} & \mathrm{~s}_{24}
\end{array}\right]_{3 \times 8} \\
\mathbf{S}_{4}=\left[\begin{array}{lllllllll}
\mathrm{s}_{1} & \mathrm{~s}_{5} & \mathrm{~s}_{9} & \mathrm{~s}_{13} & \mathrm{~s}_{17} & \mathrm{~s}_{21} & \mathrm{~s}_{25} & \mathrm{~s}_{29} \\
\mathrm{~s}_{2} & \mathrm{~s}_{6} & \mathrm{~s}_{10} & \mathrm{~s}_{14} & \mathrm{~s}_{18} & \mathrm{~s}_{22} & \mathrm{~s}_{26} & \mathrm{~s}_{30} \\
\mathrm{~s}_{3} & \mathrm{~s}_{7} & \mathrm{~s}_{11} & \mathrm{~s}_{15} & \mathrm{~s}_{19} & \mathrm{~s}_{23} & \mathrm{~s}_{27} & \mathrm{~s}_{31} \\
\mathrm{~s}_{4} & \mathrm{~s}_{8} & \mathrm{~s}_{12} & \mathrm{~s}_{16} & \mathrm{~s}_{20} & \mathrm{~s}_{24} & \mathrm{~s}_{28} & \mathrm{~s}_{32}
\end{array}\right]_{4 \times 8}
\end{gathered}
$$

For these simulations it was assumed that different subcarriers for the MC-CDMA system are multiplied by independent Rayleigh fading non-selective for each subcarrier and perfectly esteemed. The signals of all users are received with the same average power.

The bandwidth of our channel is equal to $1.25 \mathrm{MHZ}$. For OFDM (IFFT) the width is divided into 256 subchannels. Four subchannels are used as guard interval, the others are used as data. Duration of an OFDM symbol is $225 \mu$ s. The $20.8 \mu \mathrm{s}$ are used for the guard interval, for the removal of the intersymbol OFDM interference and $204.2 \mu \mathrm{s}$ are used for data transmission.

In the CDMA case we use also the Hadamard matrix and the same bandwith.

The results are compared in terms of bit error rate performance (BER) versus signal-to noise ratio (SNR).

At reception the MMSE algorithm optimization with reference sequences is used for each transmit antenna $i=1, \ldots, M$. The weights of vectors $\mathbf{w}$ are calculated at each iteration based on the reference sequences included in the data frames.

The MIMO channel is considered as an $M$ SIMO independent channels and therefore each SIMO beamformer will have its own sequence reference.

It is clearly seen in Figures 5 and 7 the advantage obtained with multiple beams on the general system compared to STBC without MBF. It can see that when the number of antenna increases the better response is given.

In MBF-CDMA case For $M=3$ and $N=4$ the BER is at $0.4 \times 10^{-3}$ approximately, by cons the low BER is at $0.3 \times 10^{-4}$ for $M=4$ and $N=4$.

In MC-CDMA case For $M=3$ and $N=4$ the BER is at $0.5 \times 10^{-4}$ approximately, by cons the low BER is at $10^{-5}$ and an SNR at $13 \mathrm{~dB}$ only for $M=4$ and $N=4$.

The simulation of Figures 6 and 8 shows the advantage largely contributed by multiple beamforming and VBLAST detector from ZF or MMSE, the bit error rate is at $10^{-5}$ for an SNR of $14 \mathrm{~dB}$ in the CDMA case and the BER is at $10^{-5}$ at $11 \mathrm{~dB}$ in the MC-CDMA case . In addition, the number of antennas increases the better result. Compare also the case of STBC it was a low BER and low SNR for the V-BLAST case.

It can see in this work the advantage largely provided by the MBF-MC-CDMA system over the MBF-CDMA system in frequency selective channel.

\section{CONCLUSION}

The objective of this research was combined multi-access with MIMO channels composed by antennas array at trasmitter and antenna arrays at receiver with adaptive multiple beamforming (multi-beam) at receiver. optimization criterion chosen was the Minimum mean Square Error (MMSE) algorithm for its ease of implementation using a reference sequence for each transmit antenna $i=1, \ldots, M$ in the detection of transmitted symbols for MBF-STBC-CDMA,MBF-V-BLAST-CDMA,MBF-STBCMC-CDMA and MBF-V-BLAST-MC-CDMA systems .

STBC coding has made a significant gain in terms of diversity and beamforming most strongly contributed to reduce or virtually eliminate the effect of multipath, then the vertical VBLAST architecture which makes just a demultiplexing chain 
into sub channel information, each of them being transmitted by its respective antenna $i$ after being modulated, provides a better performance compared to the STBC coding studied and therefore greater transmission capacity .

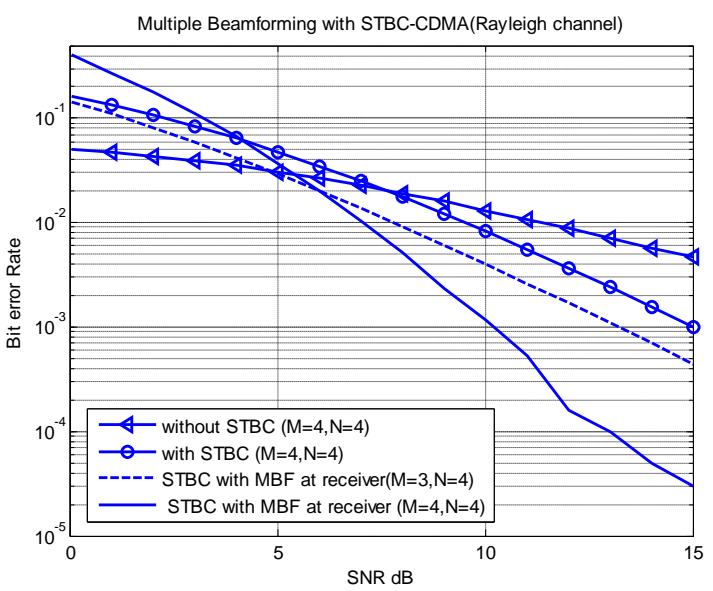

Fig 5: Performance of STBC-MBF-MIMO-CDMA

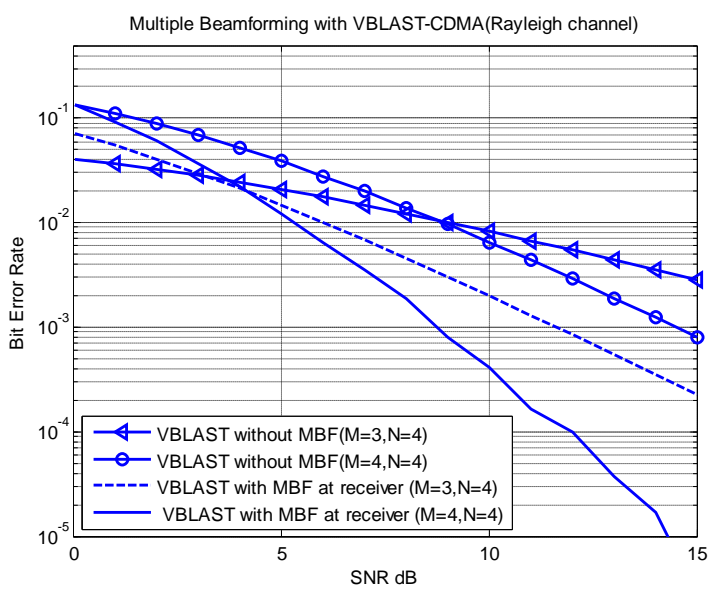

Fig 6: Performance of VBLAST-MBF-MIMO-CDMA

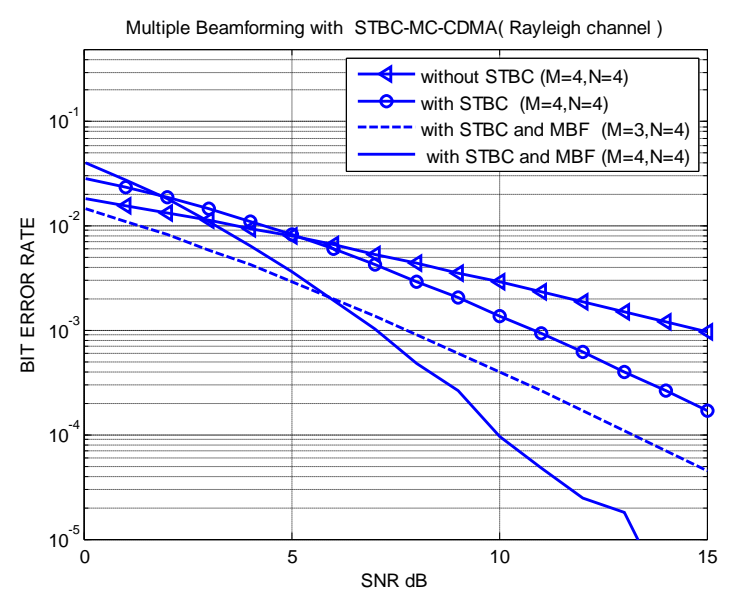

Fig 7: performance of STBC-MBF-MIMO-MC-CDMA

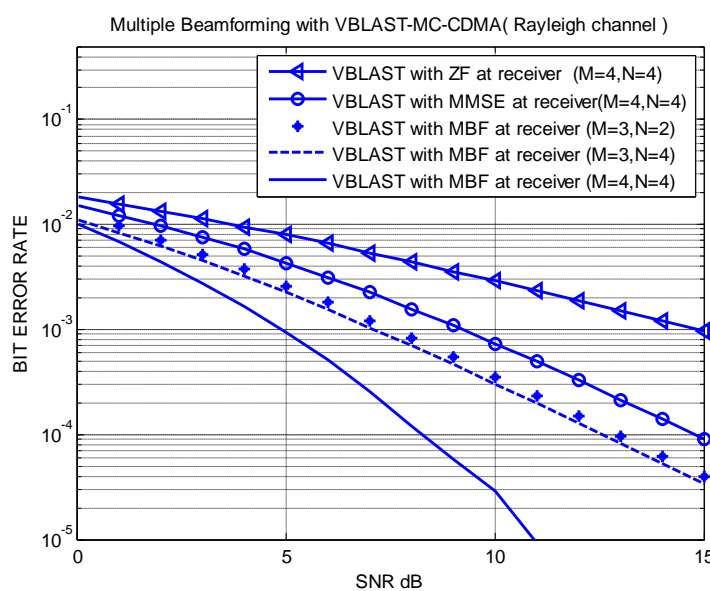

Fig 8: Performance of VBLAST-MBF-MIMO-MC-CDMA

Acronyms

BF Beamformer

BICM Bit-Interleaved Coded Modulation

MBF Multi-Beamformer

\section{REFERENCES}

[1] G. J. Foschini and M. J. Gans.'’On limits of wireless communications in a fading environment when using multiple antennas". Wireless Personal Communications, march 1998.

[2] S. Hera and R. Prasad "Overview of multicarrier CDMA," IEEE Communications Magazine, Vol. 35, No. 12, pp. 126-133, Dec.1997.

[3] E. O. J. Eriksson and V. Koivunen, "Essential statistics and tools for complex random variables," IEEE Transactions on signal processing, vol. 58, no. 10, pp. 5400-5408, October 2010.

[4] A. J. Paulraj, D. A. Gore, R. U. Nabar et H. Bolcskei, ' An overview of MIMO communications a key to gigabit wireless" Proceedings of the IEEE, vol. 2, no2, pages 198-218, f'evrier 2004.

[5] Lindner J. and Pietsch C,"The spatial dimension in the case of MC-CDMA," European Transactions on Telecommunica- tions (ETT), vol.13, pp. 431-438, Sept./Oct. 2002.

[6] V. Limpakuntorn and R. Suleesathira, "MIMO Adaptive Beamforming for MC-CDMA System," 9th International Conference on Advanced Communication Technology, pp. 2217-2221, Feb. 2007.

[7] Nevio Benvenuto, Giovanni Cherubini" Algorithms for Communications Systems and their Applications" 2002 John Wiley \& Sons Ltd.

[8] Joseph C.Liberti, Jr. Theodore S.Rappaport" Smart antennas for wireless communications" Prentice Hall PTR.

[9] B. Vucetic and J. Yuan, Space-Time Coding, Wiley, 2003. 